\title{
The Brauer-Kuroda formula for higher $S$-class numbers in dihedral extensions of number fields
}

\author{
Luca Caputo*
}

November 19, 2018

\begin{abstract}
Let $p$ be an odd prime and let $L / k$ be a Galois extension of number fields whose Galois group is isomorphic to the dihedral group of order $2 p$. Let $S$ be a finite set of primes of $L$ which is stable under the action of $\operatorname{Gal}(L / k)$. The Lichtenbaum conjecture on special values of the Dedekind zeta function at negative integers, together with Brauer formalism for Artin's $L$-functions, gives a (conjectural) formula relating orders of motivic cohomology groups of rings of $S$-integers and higher regulators of the subextensions of $L / k$. In analogy with the classical case of special values at 0 , we give an algebraic proof of this formula, i.e. without using the Lichtenbaum conjecture nor Brauer formalism. Our method also gives an interpretation of the regulator term as a higher unit index.
\end{abstract}

2000 Mathematical Subject Classification: Primary 19F27 Secondary $11 R 20$

\section{General setting and statement of the main result}

For a number field $E$ and a finite set of places $S$ of $E$, denote by $\zeta_{E}^{S}$ the Dedekind $S$-zeta function of $E$. For a complex number $s$, denote by $\zeta_{E}^{S}(s)^{*}$ the special value of $\zeta_{E}^{S}$ at $s$ (i.e. the first nontrivial coefficient of the Laurent expansion of $\zeta_{E}^{S}$ around $s$ ). Then by a well-known result of Dirichlet we have the formula

$$
\zeta_{E}^{S}(0)^{*}=-\frac{h_{E}^{S}}{w_{E}} R_{E}^{S}
$$

where $h_{E}^{S}$ is the class number of the ring $\mathcal{O}_{E}^{S}$ of $S$-integers of $E, w_{E}$ is the order of the group of roots of unity of $E$ and $R_{E}^{S}$ is the regulator of $\left(\mathcal{O}_{E}^{S}\right)^{\times}$. There are conjectural analogues of this formula when 0 is replaced by negative integers: more precisely, for any integer $m \geq 2$, a $S$-version of the Lichtenbaum conjecture reads (see [Li], [Ko], [Ka]...)

$$
\zeta_{E}^{S}(1-m)^{*}=(-1)^{t_{E}, m} \frac{h_{E, m}^{S}}{w_{E, m}} R_{E, m}
$$

Here $h_{E, m}^{S}$ is the order of the motivic cohomology group $H^{2}\left(\mathcal{O}_{E}^{S}, \mathbb{Z}(m)\right.$ ) (which is finite by the Bloch-Kato "conjecture"), $w_{E, m}$ is the order of the torsion subgroup of the motivic cohomology group $H^{1}\left(\mathcal{O}_{E}^{S}, \mathbb{Z}(m)\right)$ (which is a finitely generated $\mathbb{Z}$-module by the Bloch-Kato "conjecture") and $R_{E, m}$ is the (motivic) regulator of $H^{1}\left(\mathcal{O}_{E}^{S}, \mathbb{Z}(m)\right.$ ) (see Section 3). In this paper we use the definition of motivic cohomology in terms of Bloch's higher Chow groups, in other words

$$
H^{j}\left(\mathcal{O}_{E}^{S}, \mathbb{Z}(m)\right):=C H^{m}\left(\operatorname{Spec}\left(\mathcal{O}_{E}^{S}\right), 2 m-j\right)
$$

Finally, $t_{E, m} \in \mathbb{N}$ is given by

$$
t_{E, m}=\left\{\begin{array}{cc}
1 & \text { if } m \equiv 1 \bmod 4 \\
r_{1}(E)+r_{2}(E) & \text { if } m \equiv 2 \bmod 4 \\
r_{1}(E) & \text { if } m \equiv 3 \bmod 4 \\
r_{2}(E) & \text { if } m \equiv 0 \bmod 4
\end{array}\right.
$$

\footnotetext{
${ }^{*}$ Supported by an IRCSET fellowship.
} 
where $r_{1}(E)$ (resp. $r_{2}(E)$ ) is the number of real places (resp. complex places) of $E$.

We now list some known facts about the Lichtenbaum conjecture and motivic cohomology: for any further detail and reference to relevant literature we refer the reader to Kolster's and Kahn's excellent surveys ([Ko] and $[\mathrm{Ka}]$ ). Here we just recall that, if $m \geq 2$, we have

$$
H^{1}\left(\mathcal{O}_{E}^{S}, \mathbb{Z}(m)\right) \otimes \mathbb{Q} \cong K_{2 m-1}\left(\mathcal{O}_{E}^{S}\right) \otimes \mathbb{Q}
$$

and, since the Bloch-Kato conjecture on the Galois symbol holds (see [We]), we also have

$$
H^{j}\left(\mathcal{O}_{E}^{S}, \mathbb{Z}(m)\right) \otimes_{\mathbb{Z}} \mathbb{Z}_{\ell} \cong H_{e ́ t}^{j}\left(\mathcal{O}_{E}^{S}\left[\frac{1}{\ell}\right], \mathbb{Z}_{\ell}(m)\right) \quad j=1,2
$$

where $\ell$ is any prime. Whenever a Galois action is defined on $E$, the above isomorphisms are invariant under this action.

Then thanks to Wiles' proof of the main conjecture in Iwasawa theory, the Lichtenbaum conjecture is known for $m \geq 2$ even and $E$ totally real abelian and it is also known to hold up to power of 2 for $m \geq 2$ even and $E$ totally real. More generally it is known to hold up to power of 2 for $E$ abelian and any $m \geq 2$.

Note that $H^{1}\left(\mathcal{O}_{E}^{S}, \mathbb{Z}(m)\right)$ does not depend on $S$ (use for example (4) and the correspondig property for étale cohomology groups, see Lemma 2.10): so we shall omit the reference to $S$ in $w_{E, m}^{S}$ and $R_{E, m}^{S}$.

Now let $p$ be an odd prime. Let $D=D_{p}$ denote the dihedral group of order $2 p$ : in particular

$$
D=\left\langle\tau, \sigma \mid \tau^{p}=\sigma^{2}=1, \sigma \tau \sigma=\tau^{-1}\right\rangle
$$

Let $L / k$ be a Galois extension of number fields such that $\operatorname{Gal}(L / k) \cong D$ (in the rest of this paper we shall identify those groups). Let $K$ (resp. $K^{\prime}$ ) be the subfield of $L$ fixed by $\langle\sigma\rangle$ (resp. by $\left\langle\tau^{2} \sigma\right\rangle$ ): in particular $K^{\prime}=\tau(K)$. Let $F$ be the subfield of $L$ fixed by $\langle\tau\rangle$ : set $G=\operatorname{Gal}(L / F)$ and $\Delta=\operatorname{Gal}(F / k)$.

Let $S$ be a finite set of places of $L$ which is stable under the action of $D$ and contains the archimedean primes (we shall consider only sets of primes containing the archimedean ones, so we will not further mention this property). For any subfield $E$ of $L$ containing $k$, the set of places of $E$ which lie below those of $S$ will be denoted by $S_{E}$ or simply again by $S$ if no misunderstanding is possible. The existence of the nontrivial $D$-relation (in the sense of [DD], see Definition 2.1 and Example 2.4 of that paper)

$$
\{1\}-2\langle\sigma\rangle-G+2 D
$$

together with Brauer formalism for Artin's $L$-functions, gives the following formula

$$
\zeta_{L}^{S}(s)=\zeta_{F}^{S}(s) \frac{\zeta_{K}^{S}(s)^{2}}{\zeta_{k}^{S}(s)^{2}}
$$

Considering the special value at 0 of (66) and using (11), we get

$$
h_{L}^{S}=h_{F}^{S} \frac{\left(h_{K}^{S}\right)^{2}}{\left(h_{k}^{S}\right)^{2}} \cdot \frac{w_{k}^{2} w_{L}}{w_{K}^{2} w_{F}} \cdot \frac{\left(R_{K}^{S}\right)^{2} R_{F}^{S}}{\left(R_{k}^{S}\right)^{2} R_{L}^{S}}
$$

which is commonly referred to as the (classical) Brauer-Kuroda formula (for dihedral extensions of order $2 p$ ). It can be shown that the $w$-factor is actually trivial. More interestingly, the factor involving regulators and roots of unity can be expressed as an index of subgroups whose definition involves units of subextensions of $L / k$ (see [Ba], $[\mathrm{Ja},[\mathrm{HK}], \mathrm{Lem}, \ldots$ ). Considering special values at negative integers and using (2), we get of course, for any $m \geq 2$, a conjectural analogue of (7)

$$
h_{L, m}^{S}=h_{F, m}^{S} \frac{\left(h_{K, m}^{S}\right)^{2}}{\left(h_{k, m}\right)^{2}} \cdot \frac{\left(w_{k, m}^{S}\right)^{2} w_{L, m}^{S}}{\left(w_{K, m}^{S}\right)^{2} w_{F, m}^{S}} \cdot \frac{\left(R_{K, m}^{S}\right)^{2} R_{F, m}^{S}}{\left(R_{k, m}^{S}\right)^{2} R_{L, m}^{S}}
$$


(it is easy to see that indeed the signs appearing in (2) cancel each other out in (8), use for example Lemma 2.37 of $[\mathrm{DD}]$ ).

In this paper we prove (8) without using the Lichtenbaum conjecture and actually in an algebraic way, i.e. we make no use of $L$-functions at all. It is worth noting that the $w$-term in the above formula is trivial, as in the classical case, thanks to the following lemma (if $A$ is a ring and $M$ is an $A$-module, $\operatorname{tor}_{A}(M)$ denotes the torsion submodule of $M$ ).

Lemma 1.1. Let $S$ be a finite set of places of $L$ which is invariant under the action of $\mathrm{Gal}(L / k)$. Then

$$
w_{L, m}=w_{F, m} \quad \text { and } \quad w_{K, m}=w_{k, m}
$$

Proof. Recall that for any number field $E$ and any prime $\ell$, we have $(m \geq 2)$

$$
\operatorname{tor}_{\mathbb{Z}}\left(H^{1}\left(\mathcal{O}_{E}^{S}, \mathbb{Z}(m)\right)\right) \otimes_{\mathbb{Z}} \mathbb{Z}_{\ell} \cong \operatorname{tor}_{\mathbb{Z}_{\ell}}\left(H_{e ́ t}^{1}\left(\mathcal{O}_{E}^{S}\left[\frac{1}{\ell}\right], \mathbb{Z}_{\ell}(m)\right)\right) \cong H^{0}\left(E, \mathbb{Q}_{\ell} / \mathbb{Z}_{\ell}(m)\right)
$$

and the latter has cardinality $v_{\ell}\left(\kappa_{E}\left(\gamma_{E}\right)^{m}-1\right)$, where $v_{\ell}$ is the $\ell$-adic valuation such that $v_{\ell}(\ell)=1$, $\kappa_{E}: \Gamma_{E} \rightarrow \mathbb{Z}_{\ell}^{\times}$is the cyclotomic character evaluated on $\Gamma_{E}=\operatorname{Gal}\left(E\left(\mu_{\ell}\right) / E\right)$ and $\gamma_{E}$ is any topological generator of $\Gamma_{E}$. Now, since $L / k$ is not abelian, $L \cap F\left(\mu_{\ell \infty}\right)=F$. This shows that

$$
v_{\ell}\left(\kappa_{L}\left(\gamma_{L}\right)^{m}-1\right)=v_{\ell}\left(\kappa_{F}\left(\gamma_{F}\right)^{m}-1\right)
$$

since restriction maps $\Gamma_{L}$ isomorphically onto $\Gamma_{F}$. A similar argument apply for $K$ and $k$.

The proof of the conjectural formula above is achieved by summing up the following two results (which are proved respectively in Section 2 and Section 3), which are maybe interesting in their own right.

Formula 1. The following formula holds

$$
h_{L, m}^{S}=p^{-\alpha_{m}} h_{F, m}^{S}\left(\frac{h_{K, m}^{S}}{h_{k, m}^{S}}\right)^{2} u_{m},
$$

where $\alpha_{m}=\operatorname{rk}_{\mathbb{Z}_{p}} H_{F, m}-\operatorname{rk}_{\mathbb{Z}_{p}} H_{k, m}$ and

$$
u_{m}=\frac{\left(H_{L, m}: H_{F, m} H_{K, m} H_{K^{\prime}, m}\right)\left(\left(\bar{H}_{F, m}\right)^{\Delta}: \bar{H}_{k, m}\right)}{\left(\left(\bar{H}_{F, m}\right)^{D}: \bar{H}_{k, m}\right)}
$$

where, for a number field $E$, we have set $H_{E, m}=H^{1}\left(\mathcal{O}_{E}^{S}, \mathbb{Z}_{p}(m)\right)$ and $\bar{H}_{E, m}=H_{E, m} /$ tor $_{\mathbb{Z}} H_{E, m}$.

Formula 2. With notation as in the previous formula, the following formula holds

$$
p^{-\alpha_{m}} u_{m}=\frac{\left(R_{K, m}\right)^{2} R_{F, m}}{\left(R_{k, m}\right)^{2} R_{L, m}}
$$

Note that indeed $u_{m}$ and $\alpha_{m}$ do not depend on $S$ because $H_{E, m}$ doesn't. It will turn out that, as in the classical case, $u_{m}$ is a power of $p$.

We will divide the proof of Formula 1 in two parts, studying separatedly $p$-parts and $\ell$-parts for any prime $\ell \neq p$ (using then (4) to glue all parts together). It should be stressed that the proof for $\ell$-parts with $\ell \neq p$ is really much easier: using just the fact that the cohomology groups involved are cohomological Mackey functors (in the sense of Dress, see for example $[\mathrm{BO}]$ ) and that $D=D_{p}$ is not $\ell$-hypoelementary, we get even more precise structural relations. The proof for $p$-parts uses mainly descent and co-descent results for étale cohomology groups (which are described in [KM], see also [Ko]). This is essentially the only arithmetic information which is needed, the rest of the proof being a technical algebraic computation. The 
proof of Formula 1 given here can probably be generalized without too much effort to metabelian groups whit commutators subgroup of order a power of $p$ and index coprime with $p$. However that seems not to be the best approach for the general case of an arbitrary Galois group. We believe anyway that there exists a general algebraic proof of Brauer-Kuroda formulas for an arbitrary finite Galois extension (a higher analogue of $[\mathrm{dS}]$ ). It is worth noting that any proof of the classical version of Formula 1 (see [Ja], [HK], $[\mathrm{Lem}, \ldots)$ uses class field theory and genus theory and considers $p$-parts and $\ell$-parts for $\ell \neq p$ separatedly.

In the last section we perform the proof of Formula 2. The translation of $u_{m}$ in terms of higher (or motivic) regulators is done using methods from representation theory which have been introduced by the Dokchitser brothers (see for example [DD]). In particular we follow the strategy of Bartel (see [Ba]), who used Dokchitser's ideas to prove a statement analogous to ours in the classical case. In order to use these techniques, we need a higher version of Dirichlet's theorem on the Galois structure of units, which we state and prove, since we could not find it in the literature.

\section{Notation and standard results}

- As before, throughout the paper, if $A$ is a commutative ring and $M$ is an $A$-module, $\operatorname{tor}_{A}(M)$ denotes the torsion submodule of $M$. We will also use the notation $\bar{M}$ for $M / \operatorname{tor}_{A} M$ without any specific mention to $A$, since it will be clear from the context which is the ring we are considering. Finally, for any $a \in A$, we set $M[a]=\{m \in M \mid a m=0\}$.

- Let $H$ be a finite group. We denote by $N_{H}=\sum_{h \in H} h \in A[H]$ the norm element and by $I_{H} \subseteq A[H]$ the augmentation ideal. If $B$ is a $A[H]$-module, we use the following notation

$$
\begin{aligned}
& -B^{H}=\{b \in B \mid h b=b \text { for all } h \in H\} \\
& -B_{H}=B / I_{H} B \\
& -B\left[N_{H}\right]=\left\{b \in B \mid N_{H} b=0\right\} .
\end{aligned}
$$

If $\ell$ is a prime, $A=\mathbb{Z}_{\ell}$ and $H$ is a $q$-group for some prime $q \neq \ell$, then

$$
B^{H}=N_{H} B \quad \text { and } \quad B\left[N_{H}\right]=I_{H} B
$$

since $B$ is $q$-divisible (being a $\mathbb{Z}_{\ell}$-module) and hence $H$-cohomologically trivial.

Aknowledgements I would like to thank Kevin Hutchinson for many enlightening discussions on this subject and Manfred Kolster for making me aware of the work of the Dokchitser brothers.

\section{A formula relating higher class numbers and a higher units index}

In this section we prove Formula 1, First we study the $p$-part of the problem which is the most delicate. The natural number $m \geq 2$ will be fixed throughout the section. For any number field $E$ such that $k \subseteq E \subseteq L$ and any finite set $S$ of primes of $L$, we set

$$
\begin{aligned}
& U_{E, m}=H_{e ́ t}^{1}\left(\mathcal{O}_{E}^{S}\left[\frac{1}{p}\right], \mathbb{Z}_{p}(m)\right) \\
& A_{E, m}^{S}=H_{e ́ t}^{2}\left(\mathcal{O}_{E}^{S}\left[\frac{1}{p}\right], \mathbb{Z}_{p}(m)\right)
\end{aligned}
$$

(in fact, $U_{E, m}$ does not depend on $S$, see Lemma 2.10) We also fix for this section a finite set $T$ of primes of $L$ such that

- $T$ is stable under the action of $D=\operatorname{Gal}(L / k)$;

- $T$ contains those primes which ramify in $L / k$;

- $T$ contains all the primes above $p$. 
Since $T$ and $m$ are fixed for this section we will also use the notation $U_{E}$ for $U_{E, m}$ and $A_{E}$ for $A_{E, m}^{T}$. Both $A_{E}$ and $U_{E}$ are abelian groups: however, because of their analogies with the ideal class group and the unit group of $E$ respectively, we are going to use multiplicative notation for them.

Note that if $Q$ is a group of automorphisms of $E$ of order 2, then $U_{E}^{Q}=U_{E^{Q}}$ and $A_{E}^{Q}=A_{E^{Q}}$ : this follows from the fact that $\mathbb{Z}_{p}(m)$ is $Q$-cohomologically trivial. The following well-known result gives us the description of $G$-descent for $U_{E}$ and $A_{E}$ (recall that $G=\operatorname{Gal}(L / F)$ ).

Proposition 2.1. The natural map $U_{F} \rightarrow U_{L}^{G}$ is an isomorphism and we have an exact sequence

$$
0 \rightarrow H^{1}\left(G, U_{L}\right) \rightarrow A_{F} \rightarrow A_{L}^{G} \rightarrow H^{2}\left(G, U_{L}\right) \rightarrow 0
$$

Proof. See $[\mathrm{KM}]$, Theorem 1.2. Note that the hypotheses are satisfied thanks the properties we required on $T$.

We will often identify $U_{F}$ with its image in $U_{L}$ (the same will be done with $U_{K}, U_{K}^{\prime}$ and $U_{k}$ ). In the same way, we will identify $A_{K}$ and $A_{K^{\prime}}$ with their images in $A_{L}$. We record now the following easy lemma which will be used repeatedly for the rest of this section.

Lemma 2.2. Let $M$ be a 2-divisible D-module: then the Tate isomorphisms $\widehat{H}^{j}(G, M) \cong \widehat{H}^{j+2}(G, M)$ are $\Delta$-antiequivariant, so that in particular, if $H^{j}(G, M)$ is finite for any $j \in \mathbb{Z}$, we have

$$
\left|\widehat{H}^{j}(D, M)\right|=\left|\widehat{H}^{j+2}(G, M)\right| /\left|\widehat{H}^{j+2}(D, M)\right|
$$

Proof. We only need to show that Tate's isomorphism is $\Delta$-antiequivariant. Recall that the Tate isomorphism is given by the cup product with a fixed generator $\chi$ of $H^{2}(G, \mathbb{Z})$ :

$$
\begin{array}{ccc}
\widehat{H}^{i}(G, M) & \longrightarrow & \widehat{H}^{i+2}(G, M) \\
x & \longmapsto & x \cup \chi
\end{array}
$$

The action of $\delta \in \Delta$ on $\widehat{H}^{i}(G, M)$ is $\delta_{*}$ in the notation of [NSW], I.5 and this action is -1 on $H^{2}(G, \mathbb{Z})$ as can immediately be seen through the isomorphism $H^{2}(G, \mathbb{Z}) \cong H^{1}(G, \mathbb{Q} / \mathbb{Z})=\operatorname{Hom}(G, \mathbb{Q} / \mathbb{Z}$ ) (which comes from the exact sequence $0 \rightarrow \mathbb{Z} \rightarrow \mathbb{Q} \rightarrow \mathbb{Q} / \mathbb{Z} \rightarrow 0$ and the fact that $\mathbb{Q}$ is $G$-cohomologically trivial being $p$-divisible). Then, by Proposition 1.5.3 of [NSW], $\delta_{*}(x \cup \chi)=-\left(\delta_{*} x\right) \cup \chi$ which gives the result.

The next lemma deals with the subgroup $A_{K} A_{K^{\prime}} \subseteq A_{L}$ but there is an analogous version for $U_{K} U_{K^{\prime}} \subseteq$ $U_{L}$ (just replace $A$ by $U$ in the statement).

Lemma 2.3. The subgroup $A_{K} A_{K^{\prime}} \subseteq A_{L}$ is a $D$-module and

$$
A_{K} A_{K^{\prime}}=\prod_{j=0}^{p-2} A_{K}^{\tau}=\prod_{j=0}^{p-2} A_{\tau(K)}
$$

Moreover $I_{G} A_{L} \subseteq A_{K} A_{K^{\prime}}$.

Proof. For the first assertion, see [HK], Lemma 1. For the last one, see [Lem], Lemma 3.3.

We now start with the proof of the $p$-part of Formula 1 .

Lemma 2.4. Define $\iota: A_{K} \oplus A_{K^{\prime}} \rightarrow A_{L}$ as $\iota\left(a, a^{\prime}\right)=a a^{\prime}$. Then there is an exact sequence as follows

$$
0 \rightarrow H^{0}\left(D, A_{L}\right) \rightarrow A_{K} \oplus A_{K^{\prime}} \stackrel{\iota}{\rightarrow} A_{L} \rightarrow H_{0}\left(G, A_{L}\right) / H_{0}\left(G, A_{L}\right)^{\Delta} \rightarrow 0
$$

Proof. It is easy to see that the map Ker $\iota \rightarrow H^{0}\left(D, A_{L}\right)$ given by $\left(a, a^{\prime}\right) \mapsto a$ is indeed an isomorphism. As for the cokernel of $\iota$, note that $I_{G} A_{L} \subseteq A_{K} A_{K^{\prime}}$ by Lemma 2.3. Now the claim follows since

$$
A_{K^{\prime}} I_{G} A_{L} / I_{G} A_{L}=\left(A_{L} / I_{G} A_{L}\right)^{\left\langle\tau^{2} \sigma\right\rangle}=\left(A_{L} / I_{G} A_{L}\right)^{\langle\sigma\rangle}=A_{K} I_{G} A_{L} / I_{G} A_{L}
$$

Therefore

$$
H_{0}\left(G, A_{L}\right)^{\Delta}=\left(A_{L} / I_{G} A_{L}\right)^{\Delta}=A_{K} A_{K^{\prime}} I_{G} A_{L} / I_{G} A_{L}=A_{K} A_{K^{\prime}} / I_{G} A_{L}
$$


Lemma 2.5. The following equality holds

$$
\left|H^{0}\left(D, A_{L}\right)\right|=\frac{\left|H^{2}\left(D, U_{L}\right)\right| \cdot\left|A_{k}\right|}{\left|H^{1}\left(D, U_{L}\right)\right|}
$$

Proof. Take $\Delta$-invariants of the exact sequence in Proposition 2.1; the sequence stays exact. Then use that $\left(A_{L}^{G}\right)^{\Delta}=A_{L}^{D}, A_{F}^{\Delta} \cong A_{k}$ and $H^{j}\left(G, U_{L}\right)^{\Delta}=H^{j}\left(D, U_{L}\right)(j=1,2)$ because $U_{L}^{G}=U_{F}$ is $\Delta$-cohomologically trivial (being 2-divisible).

The next lemma describe codescent for $A_{L}$.

Lemma 2.6. The corestriction map induces isomorphisms $H_{0}\left(G, A_{L}\right) \cong A_{F}$ and $H_{0}\left(G, A_{L}\right)^{\Delta} \cong A_{k}$.

Proof. For the first isomorphism use [KM], Proposition 1.3. Then note that the second isomorphism follows from the first one being $\Delta$-equivariant (since corestriction commutes with conjugation).

In what follows we shall rewrite the orders of $H^{1}\left(D, U_{L}\right)$ and $H^{2}\left(D, U_{L}\right)$ in terms of certain unit indexes. We first quote a simple lemma which has been used already by Lemmermeyer (see [Lem], Section 5).

Lemma 2.7. Let $f: B \rightarrow B^{\prime}$ be a homomorphism of abelian groups and let $C$ be a subgroup of finite index in $B$. Then

$$
(B: C)=(f(B): f(C)) \cdot(\operatorname{Ker} f: \operatorname{Ker} f \cap C)
$$

Proof. This is clear because we have the exact sequence

$$
0 \rightarrow(C+\operatorname{Ker} f) / C \rightarrow B / C \rightarrow f(B) / f(C) \rightarrow 0
$$

Remark. The preceding lemma implies in particular the following equality $\left(B=B^{\prime}=U_{L}, f=N_{G}\right)$

$$
\left(U_{L}: U_{K} U_{K^{\prime}} U_{F}\right)=\left(N_{G} U_{L}: N_{G}\left(U_{K} U_{K^{\prime}} U_{F}\right)\right) \cdot\left(U_{L}\left[N_{G}\right]: U_{L}\left[N_{G}\right] \cap U_{K} U_{K^{\prime}} U_{F}\right)
$$

Note that $U_{K} U_{K^{\prime}} U_{F}$ is of finite index in $U_{L}$ because, for example, $N_{G}\left(U_{K} U_{K^{\prime}} U_{F}\right)$ is of finite index in $N_{G} U_{L}$ (since both are of finite index in $U_{F}=U_{L}^{G}$ ) and $U_{L}\left[N_{G}\right] \cap U_{K} U_{K^{\prime}} U_{F}$, which contains $I_{G} U_{L}$ by Lemma 2.3. is of finite index in $U_{L}\left[N_{G}\right]$.

Recall (see Section [1) that $M[p]$ is the submodule of the $\mathbb{Z}_{p}$-module $M$ which is killed by $p$.

Lemma 2.8. We have

$$
\left|H^{1}\left(D, U_{L}\right)\right|=\left|U_{L}\left[N_{G}\right] /\left(U_{L}\left[N_{G}\right] \cap U_{K} U_{K^{\prime}} U_{F}\right)\right| \cdot\left|I_{G} U_{L} \cdot U_{F}[p] / I_{G} U_{L} \cdot U_{k}[p]\right|
$$

and

$$
\left|H^{2}\left(D, U_{L}\right)\right|=\frac{\left|U_{F} / U_{F}^{p}\right|}{\left|U_{k} / U_{k}^{p}\right| \cdot\left|N_{G} U_{L} / N_{G}\left(U_{K} U_{K^{\prime}} U_{F}\right)\right|}
$$

Proof. We prove the first assertion. The norm map

$$
U_{L} \stackrel{1+\sigma}{\longrightarrow} N_{\langle\sigma\rangle} U_{L}=U_{L}^{\langle\sigma\rangle}=U_{K}
$$

gives a map $U_{L}\left[N_{D}\right] \rightarrow U_{L}\left[N_{G}\right] \cap U_{K} U_{K^{\prime}} U_{F}$. We consider the induced map

$$
\bar{N}: U_{L}\left[N_{D}\right] \rightarrow\left(U_{L}\left[N_{G}\right] \cap U_{K} U_{K^{\prime}} U_{F}\right) / I_{G} U_{L} \cdot U_{F}[p]
$$


Note that indeed $U_{F}[p] \subseteq U_{L}\left[N_{G}\right]$ since $N_{G}$ is raising to the $p$-th power on $U_{F}$ and $I_{G} U_{L} \subseteq U_{K} U_{K^{\prime}}$ (see Lemma 2.3). Then we claim that the sequence

$$
0 \rightarrow \operatorname{Ker} \bar{N} / I_{G} U_{L} \rightarrow U_{L}\left[N_{D}\right] / I_{G} U_{L} \stackrel{\bar{N}}{\rightarrow}\left(U_{L}\left[N_{G}\right] \cap U_{K} U_{K^{\prime}} U_{F}\right) / I_{G} U_{L} \cdot U_{F}[p] \rightarrow 0
$$

is exact. Note that indeed $I_{G} U_{L} \subseteq \operatorname{Ker} \bar{N}$ since for any $u \in U_{L}$

$$
u^{(1-\tau)(1+\sigma)}=u^{(1-\tau)} \cdot u^{\sigma\left(1-\tau^{-1}\right)} \in I_{G} U_{L}
$$

The only nontrivial thing to prove is the surjectivity of $\bar{N}$ : take $u \in U_{K}, u^{\prime} \in U_{K^{\prime}}$ and $v \in U_{F}$ such that $N_{G}\left(u u^{\prime} v\right)=1$. We can find $t, t^{\prime} \in U_{L}$ such that $t^{1+\sigma}=u$ and $\left(t^{\prime}\right)^{1+\tau^{2} \sigma}=u^{\prime}$. Then

$$
1=N_{G}\left(u u^{\prime} v\right)=N_{D}\left(t t^{\prime}\right) N_{G}(v)=N_{D}\left(t t^{\prime}\right) v^{p}
$$

In particular $v^{p} \in U_{L}^{D}=U_{k}$. Note that

$$
U_{k} \cap U_{F}^{p}=U_{k}^{p}
$$

(the surjective map $U_{F} \stackrel{p}{\rightarrow} U_{F}^{p}$ stays surjective after taking $\Delta$-invariants). Hence there exists $w \in U_{k}$ such that $v^{p}=w^{p}$, which implies $v=w v_{0}$ for some $v_{0} \in U_{F}[p]$. Therefore

$$
\bar{N}\left(t t^{\prime} w^{\frac{1}{2}}\right)=u u^{\prime} v \bmod I_{G} U_{L} \cdot U_{F}[p]
$$

and the fact that $t t^{\prime} w^{\frac{1}{2}} \in U_{L}\left[N_{D}\right]$ is exactly (9). This proves that the above short sequence is exact. Now note that $I_{D} U_{L} \subseteq \operatorname{Ker} \bar{N}$ (since $I_{G} U_{L} \subseteq \operatorname{Ker} \bar{N}$ and $\left.(1+\sigma)(1-\sigma)=0\right)$ and we have an exact sequence

$$
0 \rightarrow I_{D} U_{L} / I_{G} U_{L} \rightarrow \operatorname{Ker} \bar{N} / I_{G} U_{L} \stackrel{1+\sigma}{\rightarrow}\left(I_{G} U_{L} \cdot U_{F}[p] / I_{G} U_{L}\right)^{\Delta} \rightarrow 0
$$

Surjectivity is clear since $\left(I_{G} U_{L} \cdot U_{F}[p] / I_{G} U_{L}\right)^{\Delta}=N_{\Delta}\left(I_{G} U_{L} \cdot U_{F}[p] / I_{G} U_{L}\right)$ and not only $U_{F}[p] \subseteq U_{L}\left[N_{D}\right]$ but actually $U_{F}[p] \subseteq \operatorname{Ker} \bar{N}$. To describe the kernel, set $Y=\left\{u \in U_{L} \mid u^{1+\sigma} \in I_{G} U_{L}\right\}$ and note that $I_{G} U_{L} \subseteq Y$ and $Y / I_{G} U_{L}$ equals the kernel of (11). Now if $u \in T$, then there exists $v \in U_{L}$ such that

$$
u^{1+\sigma}=v^{1-\tau}
$$

and in particular

$$
u^{2}=v^{1-\tau} u^{1-\sigma} \in I_{D} U_{L}
$$

Then $u \in I_{D} U_{L}$ and since clearly $I_{D} U_{L} \subseteq Y$, we have in fact the equality. Moreover

$$
\begin{gathered}
\left(I_{G} U_{L} \cdot U_{F}[p] / I_{G} U_{L}\right)^{\Delta} \cong\left(U_{F}[p] / I_{G} U_{L} \cap U_{F}[p]\right)^{\Delta}= \\
=U_{k}[p] / I_{G} U_{L} \cap U_{k}[p] \cong I_{G} U_{L} \cdot U_{k}[p] / I_{G} U_{L}
\end{gathered}
$$

Then

$$
\begin{gathered}
\left(U_{L}\left[N_{G}\right]: U_{L}\left[N_{G}\right] \cap U_{K} U_{K^{\prime}} U_{F}\right)=\frac{\left(U_{L}\left[N_{G}\right]: I_{G} U_{L} \cdot U_{F}[p]\right)}{\left(U_{L}\left[N_{G}\right] \cap U_{K} U_{K^{\prime}} U_{F}: I_{G} U_{L} \cdot U_{F}[p]\right)}= \\
=\frac{\left(U_{L}\left[N_{G}\right]: I_{G} U_{L}\right)}{\left(I_{G} U_{L} \cdot U_{F}[p]: I_{G} U_{L}\right) \cdot\left(U_{L}\left[N_{D}\right]: \operatorname{Ker} \bar{N}\right)}= \\
=\frac{\left|\widehat{H}^{-1}\left(G, U_{L}\right)\right|\left(\operatorname{Ker} \bar{N}: I_{D} U_{L}\right)}{\left(I_{G} U_{L} \cdot U_{F}[p]: I_{G} U_{L}\right) \cdot\left(U_{L}\left[N_{D}\right]: I_{D} U_{L}\right)}= \\
=\frac{\left|\widehat{H}^{-1}\left(G, U_{L}\right)\right|}{\left|\widehat{H}^{-1}\left(D, U_{L}\right)\right|} \cdot \frac{\left(I_{G} U_{L} \cdot U_{k}[p]: I_{G} U_{L}\right)}{\left(I_{G} U_{L} \cdot U_{F}[p]: I_{G} U_{L}\right)}= \\
=\frac{\left|H^{1}\left(D, U_{L}\right)\right|}{\left(I_{G} U_{L} \cdot U_{F}[p]: I_{G} U_{L} \cdot U_{k}[p]\right)}
\end{gathered}
$$


by Lemma 2.2. Hence the first assertion of the lemma is proved.

We now prove the second assertion (this part is actually the same as in the proof of [Lem], Theorem 2.2).

Note that $N_{G} U_{K}=N_{D} U_{L}=N_{G} U_{K^{\prime}}$ : in particular $N_{G} U_{K} \subseteq U_{L}^{D}=U_{k}$. Therefore

$$
\begin{gathered}
\left(N_{G} U_{L}: N_{G}\left(U_{K} U_{K^{\prime}} U_{F}\right)\right)=\left(N_{G} U_{L}: U_{F}^{p} \cdot N_{G} U_{K}\right)= \\
=\frac{\left(U_{F}: U_{F}^{p} \cdot N_{G} U_{K}\right)}{\left(U_{F}: N_{G} U_{L}\right)}=\frac{\left(U_{F}: U_{F}^{p}\right)}{\left(U_{F}^{p} N_{G} U_{K}: U_{F}^{p}\right) \cdot\left(U_{F}: N_{G} U_{L}\right)}= \\
=\frac{\left(U_{F}: U_{F}^{p}\right) \cdot\left(U_{F}^{p} U_{k}: U_{F}^{p} N_{G} U_{K}\right)}{\left(U_{F}^{p} U_{k}: U_{F}^{p}\right) \cdot\left(U_{F}: N_{G} U_{L}\right)}
\end{gathered}
$$

Now

$$
\begin{gathered}
\left(U_{F}^{p} U_{k}: U_{F}^{p} N_{G} U_{K}\right)=\frac{\left(U_{F}^{p} U_{k}: U_{F}^{p}\right)}{\left(U_{F}^{p} N_{G} U_{K}: U_{F}^{p}\right)}= \\
=\frac{\left(U_{k}: U_{F}^{p} \cap U_{k}\right)}{\left(N_{G} U_{K}: U_{F}^{p} \cap N_{G} U_{K}\right)}=\frac{\left(U_{k}: U_{k}^{p}\right)}{\left(N_{G} U_{K}: U_{k}^{p}\right)}=\left(U_{k}: N_{G} U_{K}\right)
\end{gathered}
$$

using (10) and

$$
U_{k}^{p}=N_{G} U_{k} \subseteq U_{F}^{p} \cap N_{G} U_{K}=U_{F}^{p} \cap N_{D} U_{L} \subseteq U_{F}^{p} \cap U_{k}=U_{k}^{p}
$$

Therefore, using once more (10),

$$
\begin{gathered}
\left(N_{G} U_{L}: N_{G}\left(U_{K} U_{K^{\prime}} U_{F}\right)\right)=\frac{\left(U_{F}: U_{F}^{p}\right)\left(U_{k}: N_{G} U_{K}\right)}{\left(U_{k}: U_{k}^{p}\right)\left(U_{F}: N_{G} U_{L}\right)}= \\
=\frac{\left|\widehat{H}^{0}\left(D, U_{L}\right)\right|}{\left|\widehat{H}^{0}\left(G, U_{L}\right)\right|} \frac{\left(U_{F}: U_{F}^{p}\right)}{\left(U_{k}: U_{k}^{p}\right)}=\frac{\left(U_{F}: U_{F}^{p}\right)}{\left(U_{k}: U_{k}^{p}\right)\left|H^{2}\left(D, U_{L}\right)\right|}
\end{gathered}
$$

by Lemma 2.2 .

Recall (see Section 10 that $\bar{M}$ is our notation for the torsion-free quotient of the $\mathbb{Z}_{p}$-module $M$.

Lemma 2.9. We have

$$
I_{G} U_{L} \cap U_{F}[p]=I_{G} U_{L} \cap U_{F} \quad \text { and } \quad I_{G} U_{L} \cap U_{k}[p]=I_{G} U_{L} \cap U_{k}
$$

Furthermore, there is an isomorphism

$$
I_{G} U_{L} \cap U_{F} \cong \bar{U}_{L}^{G} / \bar{U}_{F}
$$

which is $\Delta$-antiequivariant. In particular it induces an isomorphism

$$
I_{G} U_{L} \cap U_{F} / I_{G} U_{L} \cap U_{k} \cong \bar{U}_{L}^{D} / \bar{U}_{F}^{\Delta}=\bar{U}_{L}^{D} / \bar{U}_{k}
$$

Proof. The first assertion follows from the fact that $G$ acts trivially on $U_{F}$ and $U_{k}$ and therefore

$$
I_{G} U_{L} \cap U_{F} \subseteq U_{L}\left[N_{G}\right] \cap U_{F} \subseteq U_{F}[p] \quad \text { and } \quad I_{G} U_{L} \cap U_{k} \subseteq U_{L}\left[N_{G}\right] \cap U_{k} \subseteq U_{k}[p]
$$

Now consider the map

$$
\phi: I_{G} U_{L} \cap U_{F} \rightarrow \bar{U}_{L}^{G} / \bar{U}_{F}
$$

defined by $\phi\left(u^{1-\tau}\right)=\bar{u} \bmod \bar{U}_{F}\left(\bar{u}\right.$ is the class of $u$ in $\left.\bar{U}_{L}\right)$. First of all, this definition does not depend on the choice of $u$ : namely, if $v^{1-\tau}=u^{1-\tau}$, then $v u^{-1} \in U_{L}^{G}=U_{F}$. Of course, the image of $\phi$ is contained in $\left(\bar{U}_{L} / \bar{U}_{F}\right)^{G}=\bar{U}_{L}^{G} / \bar{U}_{F}$ (this last equality comes from $H^{1}\left(G, \bar{U}_{F}\right)=\operatorname{Hom}\left(G, \bar{U}_{F}\right)=0$ since $\bar{U}_{F}$ is a free $\mathbb{Z}_{p}$-module with trivial $G$-action). Moreover $\phi$ is clearly a homomorphism. To see that it is injective, suppose that $\phi\left(u^{1-\tau}\right)=\overline{1} \bmod \bar{U}_{F}$. This means that there exist $\zeta \in \operatorname{tor}\left(U_{L}\right)$ and $v \in U_{F}$ such 
that $u=v \zeta$. But since $\operatorname{tor}\left(U_{L}\right)=\operatorname{tor}\left(U_{F}\right)$ by Lemma 1.1, this implies $u^{1-\tau}=1$. Hence $\phi$ is injective. To prove surjectivity, choose an element $\bar{u} \in \bar{U}_{L}^{G}$. This means $u^{\tau}=u \xi$ for some $\xi \in \operatorname{tor}\left(U_{L}\right)$. Then $u^{1-\tau} \in \operatorname{tor}\left(U_{L}\right)=\operatorname{tor}\left(U_{F}\right) \subseteq U_{F}$ and $\phi\left(u^{1-\tau}\right)=\bar{u} \bmod \bar{U}_{F}$.

The map $\phi$ is $\Delta$-antiequivariant, in other words, if $\delta$ generates $\Delta$, we have

$$
\phi\left(\left(u^{1-\tau}\right)^{\delta}\right)=\phi\left(u^{1-\tau}\right)^{-\delta}
$$

for any $u^{1-\tau} \in I_{G} U_{L} \cap U_{F}$. In fact,

$$
\left(u^{1-\tau}\right)^{\delta}=u^{\sigma(1-\tau)}=u^{\left(1-\tau^{-1}\right) \sigma}=u^{(1-\tau)\left(\sum_{i=0}^{p-2} \tau^{i}\right) \sigma}
$$

Therefore

$$
\phi\left(\left(u^{1-\tau}\right)^{\delta}\right)=\overline{u^{\left(\sum_{i=0}^{p-2} \tau^{i}\right) \sigma}} \bmod \bar{U}_{F}
$$

Hence to verify (12), we have to check that

$$
v:=u^{\left(\sum_{i=0}^{p-2} \tau^{i}\right) \sigma+\sigma} \in U_{F}=U_{L}^{G}
$$

Now

$$
\begin{gathered}
v^{1-\tau^{-1}}=u^{\left(1-\tau^{-1}\right)\left(\sum_{i=0}^{p-2} \tau^{i}\right) \sigma+\left(1-\tau^{-1}\right) \sigma}=\left(u^{1-\tau}\right)^{\sigma\left(\sum_{i=0}^{p-2} \tau^{-i}\right)+\sigma}= \\
=\left(u^{1-\tau}\right)^{(p-1) \sigma+\sigma}=\left(u^{1-\tau}\right)^{p \sigma}=1
\end{gathered}
$$

since $u^{1-\tau} \in U_{F} \cap I_{G} U_{L}$ which means that $\tau$ acts trivially on it and it has order $p$. This proves that $\phi$ is $\Delta$-antiequivariant. To get the last claim of the proposition note that

$$
\left(I_{G} U_{L} \cap U_{F}\right)^{\Delta}=I_{G} U_{L} \cap U_{F} \cap U_{k}=I_{G} U_{L} \cap U_{k}
$$

and, 2-divisible modules being $\Delta$-cohomologically trivial,

$$
\left(\bar{U}_{L}^{G} / \bar{U}_{F}\right)^{\Delta}=\bar{U}_{L}^{D} / \bar{U}_{F}^{\Delta}, \quad \bar{U}_{F}^{\Delta}=U_{k}
$$

Therefore $\phi$ induces an isomorphism

$$
I_{G} U_{L} \cap U_{F} / I_{G} U_{L} \cap U_{k} \cong \bar{U}_{L}^{D} / \bar{U}_{k}
$$

We now state and prove a lemma which will allow us to get results for finite sets which are more general than our fixed $T$.

Lemma 2.10. Let $S$ be any subset of $T$ which is stable under the action of $D$ and let $S^{\prime}$ be the union of $S$ with the set of primes above $p$ in $L$. Then, for any subfield $E$ of $L$ containing $k$,

$$
H_{e ́ t}^{1}\left(\mathcal{O}_{E}^{S}\left[\frac{1}{p}\right], \mathbb{Z}_{p}(m)\right) \cong H_{e ́ t}^{1}\left(\mathcal{O}_{E}^{T}, \mathbb{Z}_{p}(m)\right) \cong H_{e ́ t}^{1}\left(E, \mathbb{Z}_{p}(m)\right)
$$

and there is an exact sequence

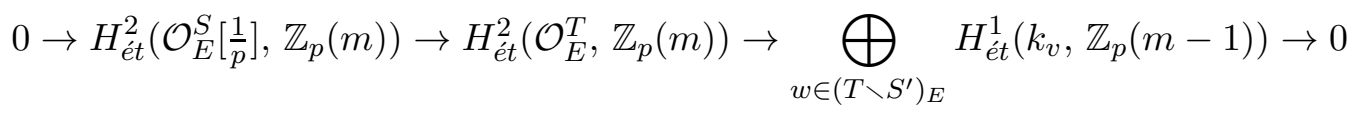

where $k_{w}$ is the residue field of $E$ at $w$. Moreover the function

$$
H \mapsto \prod_{w \in\left(T \backslash S^{\prime}\right)_{L} H}\left|H_{e ́ t}^{1}\left(k_{v}, \mathbb{Z}_{p}(m)\right)\right|
$$

which is defined on the set of subgroups of D, is trivial on D-relations (in the sense of [DD], Section 2.iii). 
Proof. The isomorphism and the exact sequence of the first part of the statement are well-known (see for example [So, Proposition 1).

As for the last part of the lemma, let $H$ be a subgroup of $D$. If $w \in\left(T \backslash S^{\prime}\right)_{L^{H}}$ and $v$ is the prime of $k$ below $w$, we have

$$
\left|\operatorname{tor} H_{e ́ t}^{1}\left(k_{w}, \mathbb{Z}_{p}(m-1)\right)\right|=\left|H_{e ́ t}^{1}\left(k_{w}, \mathbb{Z}_{p}(m-1)\right)\right|=\left|H_{e ́ t}^{0}\left(k_{w}, \mathbb{Q}_{p} / \mathbb{Z}_{p}(m-1)\right)\right|=p^{v_{p}\left(\ell_{v}^{f w(m-1)}-1\right)}
$$

where $\ell_{v}$ is the rational prime below $v, \ell_{v}^{f_{w}}=\left|k_{w}\right|$ and $v_{p}$ is the $p$-adic valuation such that $v_{p}(p)=1$. We also set $\ell_{v}^{f_{v}}=\left|k_{v}\right|$ and $f_{w \mid v}=f_{w} / f_{v}$. Then

$$
\prod_{w \in\left(T \backslash S^{\prime}\right)_{L} H}\left(\ell_{v}^{f_{w}(m-1)}-1\right)=\prod_{v \in T \backslash S^{\prime}} \prod_{j=1}^{2 p}\left(\ell_{v}^{f_{v} j(m-1)}-1\right)^{\#\left\{w \mid v \text { in } L^{H} \text { with } f_{w \mid v}=j\right\}}
$$

Using Theorem 2.36 of [DD] (as explained for instance in Example 2.37 of the same paper), we see that the function

$$
H \mapsto \prod_{w \in\left(T \backslash S^{\prime}\right)_{L^{H}}}\left(\ell_{w}^{f_{w}(m-1)}-1\right)
$$

which is defined on the set of subgroups of $D$, is trivial on $D$-relations (being a product of functions which are trivial on $D$-relations). Therefore thanks to (14), we easily see that (13) is trivial on $D$-relations.

The next proposition can be seen as the p-part of Formula 1 .

Proposition 2.11. Let $p$ be an odd prime and let $L / k$ be a Galois extension of number fields with $\operatorname{Gal}(L / k)=D$. Let $S$ be a finite set of primes of $L$ which is stable under the action of $D$. Then the following formula holds

$$
\left|A_{L, m}^{S}\right|=p^{-\alpha_{m}}\left|A_{F, m}^{S}\right| \frac{\left|A_{K, m}^{S}\right|^{2}}{\left|A_{k, m}^{S}\right|^{2}} \frac{\left(U_{L, m}: U_{K, m} U_{K^{\prime}, m} U_{F, m}\right)}{\left(\left(\bar{U}_{L, m}\right)^{D}: \bar{U}_{k, m}\right)}
$$

where $\alpha_{m}=\operatorname{rk}_{\mathbb{Z}_{p}} U_{F, m}-\operatorname{rk}_{\mathbb{Z}_{p}} U_{k, m}=\mathrm{rk}_{\mathbb{Z}} H_{F, m}-\operatorname{rk}_{\mathbb{Z}} H_{k, m}$ is as in Formula 1 .

Proof. First we prove the proposition in the case where $S=T$. Thanks to Lemma 2.9, we have

$$
\begin{gathered}
\left(I_{G} U_{L} \cdot U_{F}[p]: I_{G} U_{L} \cdot U_{k}[p]\right)=\frac{\left(I_{G} U_{L} \cdot U_{F}[p]: I_{G} U_{L}\right)}{\left(I_{G} U_{L} \cdot U_{k}[p]: I_{G} U_{L}\right)}=\frac{\left(U_{F}[p]: I_{G} U_{L} \cap U_{F}[p]\right)}{\left(U_{k}[p]: I_{G} U_{L} \cap U_{k}[p]\right)}= \\
=\frac{\left(U_{F}[p]: I_{G} U_{L} \cap U_{F}\right)}{\left(U_{k}[p]: I_{G} U_{L} \cap U_{k}\right)}=\frac{\left(U_{F}[p]: U_{k}[p]\right)}{\left(I_{G} U_{L} \cap U_{F}: I_{G} U_{L} \cap U_{k}\right)}=\frac{\left(U_{F}[p]: U_{k}[p]\right)}{\left(\bar{U}_{L}^{D}: \bar{U}_{k}\right)}
\end{gathered}
$$

Finally

$$
\frac{\left(U_{F}: U_{F}^{p}\right)}{\left(U_{k}: U_{k}^{p}\right)}=p^{\alpha_{m}}\left(U_{F}[p]: U_{k}[p]\right)
$$

where $\alpha_{m}=\operatorname{rk}_{\mathbb{Z}_{p}} U_{F, m}-\operatorname{rk}_{\mathbb{Z}_{p}} U_{k, m}=\operatorname{rk}_{\mathbb{Z}} H_{F, m}^{1}-\operatorname{rk}_{\mathbb{Z}} H_{k, m}^{1}$ (this last equality comes from (44)) is as in Formula 1. Now consider the exact sequence of Lemma 2.4 we get, using all the preceding lemmas and the remark after Lemma 2.7.

$$
\begin{gathered}
\left|A_{L, m}^{S}\right|=\frac{\left|A_{K, m}^{S}\right|^{2}\left|H_{0}\left(G, A_{L}\right)\right|\left|H^{1}\left(D, U_{L}\right)\right|}{\left|A_{k, m}^{S}\right|\left|H_{0}\left(G, A_{L}\right)^{\Delta}\right|\left|H^{2}\left(D, U_{L}\right)\right|}= \\
=\left|A_{F, m}^{S}\right| \frac{\left|A_{K, m}^{S}\right|^{2}}{\left|A_{k, m}^{S}\right|^{2}} \frac{\left(U_{L}: U_{F} U_{K} U_{K^{\prime}}\right)\left(I_{G} U_{L} \cdot U_{F}[p]: I_{G} U_{L} \cdot U_{k}[p]\right)\left(U_{k}: U_{k}^{p}\right)}{\left(U_{F}: U_{F}^{p}\right)}=
\end{gathered}
$$




$$
=p^{-\alpha_{m}}\left|A_{F, m}^{S}\right| \frac{\left|A_{K, m}^{S}\right|^{2}}{\left|A_{k, m}^{S}\right|^{2}} \frac{\left(U_{L}: U_{F} U_{K} U_{K^{\prime}}\right)}{\left(\bar{U}_{L}^{D}: \bar{U}_{k}\right)}
$$

To get the general statement, note that, by Lemma 2.10, for any subgroup $H$ of $D$, the function

$$
H \mapsto \frac{\left|A_{L^{H}, m}^{T}\right|}{\left|A_{L^{H}, m}^{S}\right|}
$$

is trivial on the relation (5).

We now deal with the general proof of Formula 1. We are going to use the language and some results of the theory of cohomological Mackey functors: instead of recalling definitions we prefer to directly refer the reader to $[\mathrm{Bo}]$, Section 1 . The next result is essentially a consequence of the fact that $D=D_{p}$ is not $\ell$-hypoelementary (a group is $\ell$-hypoelementary if it has a normal $\ell$-subgroup with cyclic quotient), provided that $\ell$ is any prime different from $p$.

Proposition 2.12. Let $\ell$ be a rational prime different from $p$. Let $S$ be a finite set of primes of $L$ which is stable under the action of $\operatorname{Gal}(L / k)$. Then there is an isomorphism of abelian groups

$$
H_{e ́ t}^{2}\left(\mathcal{O}_{L}^{S}\left[\frac{1}{\ell}\right], \mathbb{Z}_{\ell}(m)\right) \oplus H_{e ̂ t}^{2}\left(\mathcal{O}_{k}^{S}\left[\frac{1}{\ell}\right], \mathbb{Z}_{\ell}(m)\right)^{2} \cong H_{e ́ t}^{2}\left(\mathcal{O}_{F}^{S}\left[\frac{1}{\ell}\right], \mathbb{Z}_{\ell}(m)\right) \oplus H_{e ́ t}^{2}\left(\mathcal{O}_{K}^{S}\left[\frac{1}{\ell}\right], \mathbb{Z}_{\ell}(m)\right)^{2}
$$

Proof. Note that the function which assigns to any subgroup $H$ of $D$ the abelian group $H_{e t}^{2}\left(\mathcal{O}_{L^{H}}^{S}\left[\frac{1}{\ell}\right], \mathbb{Z}_{\ell}(m)\right)$ is a cohomological Mackey functor on $D$. Since $\ell \neq p, D=D_{p}$ is not $\ell$-hypoelementary which allow us to apply Theorem 1.8 of $[\mathrm{Bo}]$ to conclude.

Together with Formula 2 and a generalization of a result of Brauer (see [Ba], Theorem 5.1), the fact that $D=D_{p}$ is not $\ell$-hypoelementary if $\ell \neq p$ can also be used to give a proof of the next lemma. Here we give another proof to show that one can prove Formula 1 without using Formula 2 ,

Lemma 2.13. Let $S$ be a finite set of primes of $L$ which is stable under the action of $D=\operatorname{Gal}(L / k)$. Then the number $u_{m}$ is a power of $p:$ more precisely the following equality holds

$$
\frac{\left(U_{L, m}: U_{K, m} U_{K^{\prime}, m} U_{F, m}\right)}{\left(\left(\bar{U}_{L, m}\right)^{D}: \bar{U}_{k, m}\right)}=\frac{\left(H_{L, m}: H_{F, m} H_{K, m} H_{K^{\prime}, m}\right)\left(\left(\bar{H}_{F, m}\right)^{\Delta}: \bar{H}_{k, m}\right)}{\left(\left(\bar{H}_{L, m}\right)^{D}: \bar{H}_{k, m}\right)}=u_{m}
$$

where $H_{E, m}=H^{1}\left(\mathcal{O}_{E}^{S}, \mathbb{Z}(m)\right)$ for any subfield $E$ of $L$ containing $k$.

Proof. Note that, thanks to (4), the $p$-part of the index on the right-hand side is precisely the index on the left-hand side. Then we are left to show that, for any fixed prime $\ell \neq p$,

$$
\frac{\left(V_{L}: V_{K} V_{K^{\prime}} V_{F}\right)\left(\left(\bar{V}_{F}\right)^{D}: \bar{V}_{k}\right)}{\left(\left(\bar{V}_{L}\right)^{D}: \bar{V}_{k}\right)}=1
$$

where

$$
V_{E}=H_{e ́ t}^{1}\left(\mathcal{O}_{E}^{S}\left[\frac{1}{\ell}\right], \mathbb{Z}_{\ell}(m)\right)
$$

But this is easy, using Lemma 2.7. Details are as follows: first of all

$$
\left(V_{L}: V_{K} V_{K^{\prime}} V_{F}\right)=\left(N_{G} V_{L}: N_{G}\left(V_{K} V_{K^{\prime}} V_{F}\right)\right) \cdot\left(V_{L}\left[N_{G}\right]: V_{L}\left[N_{G}\right] \cap V_{K} V_{K^{\prime}} V_{F}\right)=1
$$

since

- $N_{G}\left(V_{L}\right)=V_{L}^{G}=V_{F}$ (because $V_{L}$ and $\mathbb{Z}_{\ell}(m)$ are $G$-cohomologically trivial);

- $N_{G}\left(V_{F}\right)=V_{F}^{p}=V_{F}$ (because $G$ acts trivially on $V_{F}$ and $V_{F}$ is $p$-divisible);

- $V_{L}\left[N_{G}\right]=I_{G} V_{L} \subseteq V_{K} V_{K^{\prime}}$ (because $V_{L}$ is $G$-cohomologically trivial and an appropriate version of Lemma 2.3 holds). 
Moreover $\bar{V}_{L}^{G}=\bar{V}_{F}$ (again because $\bar{V}_{L}$ and $\mathbb{Z}_{\ell}(m)$ are $G$-cohomologically trivial), showing that (16) holds.

The proof of Formula 1 is then achieved, because (4) allows us to glue together Proposition 2.11, Proposition 2.12 and Lemma 2.13. Note that, if $H_{F, m}$ has no $p$-torsion, then $u_{m}=\left(H_{L, m}: H_{K, m} H_{K^{\prime}, m} H_{F, m}\right)$ (this follows easily from the last assertion of Lemma 2.9).

\section{Computations with regulators}

In this section we are going to prove Formula 2, translating the higher units index

$$
u_{m}=\frac{\left(H_{L, m}: H_{F, m} H_{K, m} H_{K^{\prime}, m}\right)\left(\left(\bar{H}_{F, m}\right)^{\Delta}: \bar{H}_{k, m}\right)}{\left(\left(\bar{H}_{L, m}\right)^{D}: \bar{H}_{k, m}\right)}
$$

of Formula 1 in terms of motivic (or higher) regulators, whose definition and basic properties we briefly recall (we refer the reader to $[\mathrm{Neu}], \S 1$ ). Recall from Section 1 that, if $E$ is any number fields, we have set $H_{E, m}=H^{1}\left(\mathcal{O}_{E}^{S}, \mathbb{Z}(m)\right)$.

Let $m \geq 2$ be a natural number. If $X(E)=\operatorname{Hom}(E, \mathbb{C})$ is the set of complex embeddings of $E$, the $m$-th regulator map we shall consider is a homomorphism

$$
\rho_{E, m}: H_{E, m} \longrightarrow \bigoplus_{\beta \in X(E)}(2 \pi i)^{m-1} \mathbb{R}
$$

This map is obtained by composing the natural map $H_{E, m} \rightarrow H_{E, m} \otimes \mathbb{Q}$ with the Beilinson regulator map $K_{2 m-1}\left(\mathcal{O}_{E}\right) \otimes \mathbb{Q} \rightarrow \bigoplus_{\beta \in X(E)}(2 \pi i)^{m-1} \mathbb{R}$ via the isomorphism (3). Moreover, the image of $\rho$ is contained in the subgroup of $\bigoplus_{\beta \in X(E)}(2 \pi i)^{m-1} \mathbb{R}$ which is fixed by complex conjugation:

$$
\rho_{E, m}: H_{E, m} \longrightarrow\left(\bigoplus_{\beta \in X(E)}(2 \pi i)^{m-1} \mathbb{R}\right)^{+}
$$

The kernel of $\rho$ is exactly the torsion subgroup of $H_{E, m}$ and, thanks to Borel's theorem (and the fact that Beilinson's regulator map is twice Borel's regulator map), we know that $\rho$ induces an isomorphism $H_{E, m} \otimes \mathbb{R} \cong\left(\bigoplus_{\beta \in X(E)}(2 \pi i)^{m-1} \mathbb{R}\right)^{+}$. Then the $m$-th regulator of $E$, denoted $R_{E, m}$, is the covolume of the lattice $\rho_{E, m}\left(H_{E, m}\right)$ as a subset of the real vector space $\left(\bigoplus_{\beta \in X(E)}(2 \pi i)^{m-1} \mathbb{R}\right)^{+}$. Finally, thanks to the functorial properties of the Beilinson regulator, if a Galois action is defined on $E$, then $\rho$ is invariant under this action. Then we get a generalization of Dirichlet's theorem on units (compare with [NSW], Proposition 8.6.11). First we introduce some notation: if $\Gamma$ is a finite group and $\Gamma^{\prime}$ is a subgroup of $\Gamma$, for a $\mathbb{Z}\left[\Gamma^{\prime}\right]$-module $M$, we denote by $\operatorname{Ind}_{\Gamma^{\prime}}^{\Gamma} M$ the $\mathbb{Z}[\Gamma]$-module induced by $M$. Moreover $S_{m}(E)$ denotes the set of archimedean places of $E$ (resp. non-real archimedean places of $E$ ) if $m$ is odd (resp. if $m$ is even) and, if $E^{\prime}$ is a subfield of $E, R\left(E / E^{\prime}\right)$ denotes the set of real primes of $E^{\prime}$ which becomes complex in $E$. Finally, for a place $\mathfrak{p}$ in $S_{m}(k)$, we denote by $D_{\mathfrak{p}}$ any of the decomposition groups of places above $\mathfrak{p}$ in $L$. We will use the notation $\mathbb{1}_{D_{\mathfrak{p}}}\left(\right.$ resp. $\varepsilon_{D_{\mathfrak{p}}}$ ) for the trivial $\mathbb{Z}\left[D_{\mathfrak{p}}\right]$-module (resp. for the $\mathbb{Z}\left[D_{\mathfrak{p}}\right]$ given by the sign).

Theorem 3.1. [Higher Dirichlet's Theorem] Let $E / k$ be a Galois extension of number fields with Galois group $\Gamma$. If $m \geq 2$ is odd, then there is an isomorphism of $\mathbb{Q}[\Gamma]$-modules

$$
H^{1}\left(\mathcal{O}_{E}, \mathbb{Z}(m)\right) \otimes_{\mathbb{Z}} \mathbb{Q} \cong\left(\bigoplus_{\mathfrak{p} \in S_{m}(k)} \operatorname{Ind}_{D_{\mathfrak{p}}}^{\Gamma} \mathbb{1}_{D_{\mathfrak{p}}}\right) \otimes_{\mathbb{Z}} \mathbb{Q}
$$

while, if $m \geq 2$ is even, there is an isomorphism of $\mathbb{Q}[\Gamma]$-modules

$$
H^{1}\left(\mathcal{O}_{E}, \mathbb{Z}(m)\right) \otimes_{\mathbb{Z}} \mathbb{Q} \cong\left(\bigoplus_{\mathfrak{p} \in R(E / k)} \operatorname{Ind}_{D_{\mathfrak{p}}}^{\Gamma_{1}} \varepsilon_{D_{\mathfrak{p}}} \oplus \underset{\mathfrak{p} \in S_{m+1}(k)}{\bigoplus} \operatorname{Ind}_{D_{\mathfrak{p}}}^{\Gamma_{1}} \mathbb{1}_{D_{\mathfrak{p}}}\right) \otimes_{\mathbb{Z}} \mathbb{Q}
$$


Proof. As in the classical case, it is easy to see that

$$
\left(\bigoplus_{\beta \in X(E)}(2 \pi i)^{m-1} \mathbb{R}\right)^{+} \cong \begin{cases}\left(\bigoplus_{\mathfrak{p} \in S_{m}(k)} \operatorname{Ind}_{D_{\mathfrak{p}}}^{\Gamma} \mathbb{1}_{D_{\mathfrak{p}}}\right) \otimes_{\mathbb{Z}} \mathbb{R} & \text { if } m \text { is odd } \\ \left.\bigoplus_{\mathfrak{p} \in R(E / k)} \operatorname{Ind}_{D_{\mathfrak{p}}}^{\Gamma} \varepsilon_{D_{\mathfrak{p}}} \oplus \bigoplus_{\mathfrak{p} \in S_{m+1}(k)} \operatorname{Ind}_{D_{\mathfrak{p}}}^{\Gamma} \mathbb{1}_{D_{\mathfrak{p}}}\right) \otimes_{\mathbb{Z}} \mathbb{R} & \text { if } m \text { is even }\end{cases}
$$

as $\mathbb{R}[\Gamma]$-modules (here $S_{m+1}(k)$ is just a notation trick for the set of complex places of $k$ when $m$ is odd). The proof of the theorem then follows by the fact that $\rho_{E, m}$ is a $\mathbb{R}[\Gamma]$-isomorphism as explained above, together with Lemma 8.6.10 in [NSW].

To translate $u_{m}$ in terms of regulators, we are going to use the technique of regulators costants, introduced by the Dokchitser brothers. We first define a scalar product on $H_{E, m}$ in the following way: denote by $\langle-,-\rangle_{\infty}$ the standard scalar product on $\mathbb{C}^{|X(E)|}$. Then, for $u, v \in H_{E, m}$ we set

$$
\langle u, v\rangle_{E, m}=\left\langle\rho_{E, m}(u), \rho_{E, m}(v)\right\rangle_{\infty}
$$

It is immediate to see that $\langle-,-\rangle_{E, m}$ is a $\mathbb{Z}$-bilinear map on $H_{E, m} \times H_{E, m}$ which takes values in $\mathbb{R}$. Moreover if $E / E^{\prime}$ is a finite extension and $u, v \in H_{E^{\prime}, m}$, then

$$
\langle u, v\rangle_{E, m}=\left[E: E^{\prime}\right]\langle u, v\rangle_{E^{\prime}, m}
$$

Furthermore, if $E / E^{\prime}$ is Galois, then $\langle-,-\rangle_{E, m}$ is invariant with respect to the Galois action. The regulator map being trivial on $\operatorname{tor}_{\mathbb{Z}} H_{E, m},\langle-,-\rangle_{E, m}$ defines a $\mathbb{Z}$-bilinear map on $\bar{H}_{E, m}$.

Next we recall the definition of the regulator (or Dokchitser) constant in our particular case (see [DD], Definition 2.13 and Remark 2.27 or [Ba], Definition 2.5).

Definition 3.2. Let $M$ be a $\mathbb{Z}[D]$-module which is $\mathbb{Z}$-free of finite rank and such that $M \otimes \mathbb{Q}$ is self-dual. Let $\langle\cdot, \cdot\rangle$ be a $D$-invariant non-degenerate $\mathbb{Z}$-bilinear pairing with values in $\mathbb{R}$. Then the regulator constant of $M$ is

$$
\mathcal{C}(M)=\frac{\operatorname{det}(\langle-,-\rangle) \operatorname{det}\left(\frac{1}{|D|}\langle-,-\rangle_{\left.\right|_{M D}}\right)^{2}}{\operatorname{det}\left(\frac{1}{|G|}\langle\cdot, \cdot\rangle_{\left.\right|_{M} G}\right) \operatorname{det}\left(\frac{1}{\mid\langle\sigma\rangle}\langle-,-\rangle_{\left.\right|_{M}\langle\sigma\rangle}\right)^{2}} \in \mathbb{Q}^{\times}
$$

We will be interested in the case where $M=\bar{H}_{L, m}$ and we will use $\langle-,-\rangle_{L, m}$ to compute its regulator constant. Proposition 3.3 below shows that $\langle-,-\rangle_{L, m}$ is non-degenerate, $R_{L, m}$ being non-zero if $\bar{H}_{L, m} \neq 0$ (note also that the fact that $\bar{H}_{L, m} \otimes \mathbb{Q}$ is self-dual is equivalent to the existence of a $D$-invariant non-degenerate $\mathbb{Z}$-bilinear pairing on $\left.\bar{H}_{L, m}\right)$.

For any subfield $E$ of $L$ containing $k$, set

$$
\lambda_{E, m}=\left|\operatorname{ker}\left(\bar{H}_{E, m} \hookrightarrow \bar{H}_{L, m}^{\mathrm{Gal}(L / E)}\right)\right|
$$

It is immediate to see that

$$
\lambda_{E, m}=\left|\operatorname{coker}\left(H^{1}\left(\operatorname{Gal}(L / E), \operatorname{tor}_{\mathbb{Z}} H_{E, m}\right) \rightarrow H^{1}\left(\operatorname{Gal}(L / E), H_{E, m}\right)\right)\right|
$$

and sometimes this description will be useful: for example it shows immediatly that $\lambda_{E, m}$ is well defined (i.e. the order of the above kernel is indeed finite).

The following result is similar to Lemma 2.12 and Proposition 2.15 in $\mathrm{Ba}$ ]: we sketch the proof since there are slight differences from the proof given in that paper. Recall that $r_{1}(E)\left(\right.$ resp. $\left.r_{2}(E)\right)$ is the number of real places (resp. complex places) of the number field $E$. 
Lemma 3.3. We have

$$
\operatorname{det}\left(\langle\cdot, \cdot\rangle_{E, m}\right)=(-1)^{m-1} 2^{r_{2}(E)}\left(R_{E, m}\right)^{2}
$$

Moreover

$$
\mathcal{C}\left(\bar{H}_{L, m}\right)=\left(\frac{R_{L, m} R_{k, m}^{2}}{R_{F, m} R_{K, m}^{2}} \frac{\lambda_{F, m} \lambda_{K, m}^{2}}{\lambda_{L, m} \lambda_{k, m}^{2}}\right)^{2}
$$

Proof. The determinant of

$$
\langle-,-\rangle_{\infty}:\left(\bigoplus_{\beta \in X(E)}(2 \pi i)^{m-1} \mathbb{R}\right)^{+} \times\left(\bigoplus_{\beta \in X(E)}(2 \pi i)^{m-1} \mathbb{R}\right)^{+} \rightarrow \mathbb{R}
$$

is easily seen to be $(-1)^{m-1} 2^{r_{2}(E)}$. Then the first assertion follows by well-know properties of scalar products and the definition of $R_{E, m}$.

The proof of the second claim follows from the fact that, for any subgroup $H$ of $D$,

$$
\lambda_{L^{H}, m} \operatorname{det}\left(\langle-,-\rangle_{L, m_{\left.\right|_{H^{H}, m}}}\right)=\operatorname{det}\left(\langle-,-\rangle_{L^{H}, m}\right)
$$

(this follows easily from (19) and (18), see also [Ba], Lemma 2.13 and Lemma 2.14) and the fact that the function

$$
H \mapsto(-1)^{m-1} 2^{r_{2}\left(L^{H}\right)}
$$

defined of the set of subgroups $H$ of $D$ is trivial on relations (see [DD], Example 2.37).

The preceding lemma shows that the regulator constant of $\bar{H}_{L, m}$ is related to regulators. The next one shows that it is also related to a higher unit index. First we need some notation: we denote by $\mathbb{1}$ the trivial $\mathbb{Q}[D]$-module, by $\varepsilon$ the $\mathbb{Q}[D]$-module given by the sign and by $\omega$ an irreducible $(p-1)$-dimensional $\mathbb{Q}[D]$-module (which is unique up to isomorphism). It can be checked that $K_{0}(\mathbb{Q}[D]$ ) is a torsion-free $\mathbb{Z}$-module of rank 3 which is spanned by $\mathbb{1}, \varepsilon$ and $\omega$. We denote by $\langle\cdot, \cdot\rangle$ denotes the symmetric scalar product on $K_{0}(\mathbb{Q}[D])$ for which the basis $\{\mathbb{1}, \varepsilon, \omega\}$ is orthonormal.

Lemma 3.4. [Bartel] With the notation above, we have

$$
\mathcal{C}\left(\bar{H}_{L, m}\right)^{-\frac{1}{2}}=p^{\frac{\left\langle H_{L, m} \otimes \mathbb{Q}, \mathbb{1}-\varepsilon-\omega\right\rangle}{2}}\left(\bar{H}_{L, m}: \bar{H}_{L, m}^{\langle\sigma\rangle} \bar{H}_{L, m}^{\left\langle\omega^{2} \sigma\right\rangle} \bar{H}_{L, m}^{G}\right)
$$

Proof. See [Ba], Lemma 4.8.

Lemma 3.5. The following equality holds

$$
\frac{\left(R_{K, m}\right)^{2} R_{F, m}}{\left(R_{k, m}\right)^{2} R_{L, m}}=p^{-\alpha_{m}}\left(\bar{H}_{L, m}: \bar{H}_{L, m}^{\langle\sigma\rangle} \bar{H}_{L, m}^{\left\langle\tau^{2} \sigma\right\rangle} \bar{H}_{L, m}^{G}\right) \frac{\lambda_{F, m} \lambda_{K, m}^{2}}{\lambda_{k, m}^{2}}
$$

where $\alpha_{m}=\mathrm{rk}_{\mathbb{Z}} H_{F, m}-\mathrm{rk}_{\mathbb{Z}} H_{k, m}$ is the same as in Formula 1 .

Proof. Thanks to Lemma 3.3 and Lemma 3.4, we only have to show that

$$
\left\langle H_{L, m} \otimes \mathbb{Q}, \mathbb{1}\right\rangle-\left\langle H_{L, m} \otimes \mathbb{Q}, \varepsilon\right\rangle-\left\langle H_{L, m} \otimes \mathbb{Q}, \omega\right\rangle=-2 \alpha_{m}
$$

Suppose that $\mathfrak{P}$ is an infinite prime in $L$, such that its decomposition group $D_{\mathfrak{P}}$ in $L / k$ has order 2 . Then it is not difficult to check that

$$
\operatorname{Ind}_{D_{\mathfrak{P}}}^{D} 1_{D_{\mathfrak{P}}}=1+\omega \quad \text { and } \quad \operatorname{Ind}_{D_{\mathfrak{P}}}^{D} \varepsilon_{D_{\mathfrak{P}}}=\varepsilon+\omega
$$

as $\mathbb{Q}[D]$-modules. Furthermore

$$
\operatorname{Ind}_{\{1\}}^{D} 1_{\{1\}}=1+\varepsilon+2 \omega
$$


the left-hand side being isomorphic to the $\mathbb{Q}[D]$-module corresponding to the regular representation. Denote by $r(F / k)$ the cardinality of $R(F / k)$. If $m$ is odd, by Theorem 3.1, we have

$$
H_{L, m} \otimes_{\mathbb{Z}} \mathbb{Q}=\left(r_{1}(k)+r_{2}(k)-r(F / k)\right)(1+\varepsilon+2 \omega)+r(F / k)(1+\omega)
$$

Therefore

$$
\left\langle H_{L, m} \otimes_{\mathbb{Z}} \mathbb{Q}, 1-\varepsilon-\omega\right\rangle=2\left(r(F / k)-r_{1}(k)-r_{2}(k)\right)=-2\left(\mathrm{rk}_{\mathbb{Z}} H_{F, m}-\mathrm{rk}_{\mathbb{Z}} H_{k, m}\right)
$$

On the other hand, if $m$ is even, again by Theorem 3.1, we have

$$
H_{L, m} \otimes_{\mathbb{Z}} \mathbb{Q}=r(F / k)(\varepsilon+\omega)+r_{2}(k)(1+\varepsilon+2 \omega)
$$

Therefore

$$
\left\langle H_{L, m} \otimes_{\mathbb{Z}} \mathbb{Q}, 1-\varepsilon-\omega\right\rangle=-2\left(r(F / k)+r_{2}(k)\right)=-2\left(\mathrm{rk}_{\mathbb{Z}} H_{F, m}-\mathrm{rk}_{\mathbb{Z}} H_{k, m}\right)
$$

and this concludes the proof.

In order to get the proof of Formula 2, we need to compare the right hand side of the formula of Lemma 3.5 with $u_{m}$. For this we need some result about $\lambda_{E, m}$.

Lemma 3.6. Let $Q$ be any of the subgroups of order 2 in $D$ and let $M$ be a finitely generated $\mathbb{Z}_{2}$-module on which $D$ acts. For any $j \geq 1$, the cohomological restriction induces isomorphisms

$$
H^{j}(D, M) \cong H^{j}(Q, M)
$$

Proof. For the first assertion, note that $H^{j}(D, M)$ is an abelian 2-group since $M$ is a finitely generated $\mathbb{Z}_{2}$-module. Therefore it is well-known that the restriction map

$$
H^{j}(D, M) \longrightarrow H^{j}(Q, M)
$$

is injective since $Q$ is a 2-Sylow subgroup of $D$ (see [CE], Theorem 10.1 in Chapter XII). However by the description of the image of the restriction given in $\mathrm{CE}$, Theorem 10.1 in Chapter XII, we see that the map in (20) is in fact bijective since $Q$ has trivial intersection with any of its conjugates different from $Q$ itself (in the language of [CE], any element of $H^{j}(Q, M)$ is stable).

Lemma 3.7. The following equality holds

$$
\left(\bar{H}_{L, m}: \bar{H}_{L, m}^{\langle\sigma\rangle} \bar{H}_{L, m}^{\left\langle\tau^{2} \sigma\right\rangle} \bar{H}_{L, m}^{G}\right) \frac{\lambda_{F, m} \lambda_{K, m}^{2}}{\lambda_{k, m}^{2}}=\frac{\left(H_{L, m}: H_{K, m} H_{K^{\prime}, m} H_{F, m}\right) \lambda_{K, m}}{\lambda_{k, m}}
$$

Proof. Using the notation introduced in Section 2, we have, thanks to (17) and Lemma 1.1,

$$
\left(H_{L, m}: H_{K, m} H_{K^{\prime}, m} H_{F, m}\right)=\left(U_{L, m}: U_{K, m} U_{K^{\prime}, m} U_{F, m}\right)=\left(\bar{U}_{L, m}: \bar{U}_{K, m} \bar{U}_{K^{\prime}, m} \bar{U}_{F, m}\right)
$$

With similar arguments, it is easy to prove that we also have

$$
\left(\bar{H}_{L, m}: \bar{H}_{L, m}^{\langle\sigma\rangle} \bar{H}_{L, m}^{\left\langle\tau^{2} \sigma\right\rangle} \bar{H}_{L, m}^{G}\right)=\left(\bar{U}_{L, m}: \bar{U}_{K, m} \bar{U}_{K^{\prime}, m} \bar{U}_{L, m}^{G}\right)
$$

Furthermore, $\lambda_{K, m}$ is a power of 2 and if, for a prime $\ell,\left(\lambda_{k, m}\right)_{\ell}$ denotes the exact power of $\ell$ dividing $\lambda_{k, m}$, then we have

$$
\left(\lambda_{k, m}\right)_{2}=\lambda_{K, m}
$$

(use (19) and Lemma 3.6 applied to $M=$ tor $_{\mathbb{Z}} H_{L, m} \otimes \mathbb{Z}_{2}$ and $M=H_{L, m} \otimes \mathbb{Z}_{2}$ ). Moreover $\lambda_{F, m}=1$ or $p$ (since tor $\left(U_{F, m}\right)$ is cyclic) and $\left(\lambda_{k, m}\right)_{p} \leq \lambda_{F, m}$ (this is immediate, for example use (19)). Thus we are left to check that

$$
\left(\bar{U}_{K, m} \bar{U}_{K^{\prime}, m} \bar{U}_{L, m}^{G}: \bar{U}_{K, m} \bar{U}_{K^{\prime}, m} \bar{U}_{F, m}\right)=\frac{\lambda_{F, m}}{\left(\lambda_{k, m}\right)_{p}}
$$


Note that

$$
\left(\bar{U}_{K, m} \bar{U}_{K^{\prime}, m} \bar{U}_{L, m}^{G}: \bar{U}_{K, m} \bar{U}_{K^{\prime}, m} \bar{U}_{F, m}\right)=\left(\bar{U}_{L, m}^{G}:\left(\bar{U}_{K, m} \bar{U}_{K^{\prime}, m} \bar{U}_{F, m}\right)^{G}\right)
$$

In the rest of the proof we let the subscript $m$ drop and we shall suppose that $\bar{U}_{L}^{G} \neq \bar{U}_{F}$ (otherwise (21) is trivially true).

Suppose first that $I_{G} U_{L} \cap U_{k} \neq I_{G} U_{L} \cap U_{F}$ (which is equivalent to $\lambda_{F}=\left(\lambda_{k}\right)_{p}=p$ by Lemma 2.9). Let $u \in U_{L}$ be such that the class of $[u] \in \bar{U}_{L}$ is nontrivial in $\bar{U}_{L}^{G} / \bar{U}_{F}$ : then $u^{\tau-1}=\zeta$ generates $I_{G} U_{L} \cap U_{F}$ which is cyclic of order $p$ (note that we also have $u^{\tau^{-1}-1}=\zeta^{-1}$ ). Then $u^{(1+\sigma)} \in U_{K} \subseteq U_{K} U_{K^{\prime}} U_{F}$ and

$$
\left(u^{(1+\sigma)}\right)^{(\tau-1)}=u^{(\tau-1)(1+\sigma)}=u^{\tau-1} u^{(\tau-1) \sigma}=\zeta^{1-\sigma}=\zeta^{2}
$$

because $\sigma$ acts as -1 on $I_{G} U_{L} \cap U_{F}$ (the latter being different from $I_{G} U_{L} \cap U_{k}$ by assumption). This shows at once that $[u]^{1+\sigma} \in\left(\bar{U}_{K} \bar{U}_{K^{\prime}} \bar{U}_{F}\right)^{G}$ but $[u]^{1+\sigma} \notin \bar{U}_{F}$ (since $\sigma$ acts trivially on $\bar{U}_{L}^{G} / \bar{U}_{F}$ and $[u] \notin \bar{U}_{F}$ ) and therefore $\bar{U}_{L}^{G}=\left(\bar{U}_{K} \bar{U}_{K^{\prime}} \bar{U}_{F}\right)^{G}\left(\right.$ since $\bar{U}_{L}^{G} / \bar{U}_{F}$ has order $p$ ).

Now suppose that $I_{G} U_{L} \cap U_{k}=I_{G} U_{L} \cap U_{F}$ and we have to show that $\left(\bar{U}_{K} \bar{U}_{K^{\prime}} \bar{U}_{F}\right)^{G}=\bar{U}_{F}$. First of all we observe that

$$
U_{K}^{p} \cap U_{k}=U_{k}^{p}
$$

In fact, of course $U_{K}^{p} \cap U_{k} \supseteq U_{k}^{p}$. Conversely, if $u \in U_{K}$ and $u^{p} \in U_{k}$, then $u^{p(\tau-1)}=1$. In particular $u^{\tau-1} \in I_{G} U_{L} \cap U_{L}[p]=I_{G} U_{L} \cap U_{F}=I_{G} U_{L} \cap U_{k}$ (use Lemma 1.1] and Lemma 2.91). But this means that

$$
u^{\sigma(\tau-1)}=u^{\tau-1}
$$

and since $u^{\sigma(\tau-1)}=u^{\tau^{-1}-1}$ (because $u^{\tau^{-1}-1}=u^{-\tau+1} \in U_{k}$ ), this implies that $u^{\tau^{2}}=u$. Therefore $u \in U_{F} \cap U_{K}=U_{k}$ and therefore $U_{K}^{p} \cap U_{k} \subseteq U_{k}^{p}$. Now consider an element in $\left(\bar{U}_{K} \bar{U}_{K^{\prime}} \bar{U}_{F}\right)^{G}$ : it can be written as $\left[u v^{\tau} w\right]$ with $u, v \in U_{K}$ and $w \in U_{F}$ satisfying

$$
\left(u v^{\tau} w\right)^{\tau-1} \in \operatorname{tor} U_{L}
$$

Actually $\left(u v^{\tau} w\right)^{\tau-1}=\left(u v^{\tau}\right)^{\tau-1}=\xi \in U_{k}[p]$ (thanks to $I_{G} U_{L} \cap U_{k}=I_{G} U_{L} \cap U_{F}$, Lemma 1.1 and Lemma 2.91). This implies that

$$
\frac{u^{p \tau}}{v^{p \tau}}=\frac{u^{p}}{v^{p \tau^{2}}} \in U_{K^{\prime}}
$$

In particular

$$
\left(\frac{u^{p}}{v^{p \tau^{2}}}\right)^{\tau^{2} \sigma}=\frac{u^{p}}{v^{p \tau^{2}}}
$$

A quick calculation then shows that $(u v)^{p}=(u v)^{p \tau^{2}}$. This means that $(u v)^{p} \in U_{F} \cap U_{K}=U_{k}$. Then $(u v)^{p} \in U_{K}^{p} \cap U_{k}=U_{k}^{p}$ thanks to (22). Then $u=v^{-1} z$ with $z \in U_{k}$ and therefore

$$
\left(u v^{\tau} w\right)^{\tau-1}=\left(u v^{\tau}\right)^{\tau-1}=\left(\frac{v^{\tau}}{v}\right)^{\tau-1}=\xi \in U_{k}[p]
$$

This implies that

$$
\frac{v^{p \tau}}{v^{p}} \in U_{F} \cap I_{G} U_{L}=U_{F}[p] \cap I_{G} U_{L}
$$

which means $v^{\tau} / v \in \operatorname{tor}_{\mathbb{Z}_{p}} U_{L} \subseteq U_{F}$. Then $\xi=1$ and $\left[u v^{\tau} w\right] \in \bar{U}_{F}$. This concludes the proof.

Collecting all the results we have proved so far, we get the proof of Formula 2 and therefore of the higher Brauer-Kuroda relation (8). 


\section{References}

[Ba] A. BARTEL, On Brauer-Kuroda type relations of $S$-class numbers in dihedral extensions, arXiv: 0904.2416v3 (electronic), 2009.

[Bo] R. BoltJe, Class group relations from Burnside ring idempotents, J. Number Theory 66 n. 1 (1997), 291-305.

[CE] H. Cartan and S. Eilenberg, Homological Algebra, Princeton University Press, 1956.

[DD] T. Dokchitser And V. Dokchitser, Regulator constants and the parity conjecture, Invent. Math. $178 \mathrm{n} .1$ (2009), 23-71.

[dS] B. DE Smit, An algebraic proof of Brauer's class number relations, unpublished (an electronic copy can be found at http://www.math.leidenuniv.nl/ $\sim \operatorname{desmit} / \mathrm{res} /$ ).

[HK] F. Halter-Koch, Einheiten und Divisorenklassen in Galois'schen algebraischen Zahlkörpern mit Diedergruppe der Ordnung $2 \ell$ für eine ungerade Primzahl $\ell$, Acta Arith. XXXIII (1977), 355-364.

[Ja] J.-F. JAulent, Unités et classes dans les extensions métabeliénnes de degré n $\ell^{s}$ sur un corps de nombres algébriques, Ann. Inst. Fourier (Grenoble) 31 n. 1 (1981), 39-62.

[Ka] B. KAhn, Algebraic K-theory, algebraic cycles and arithmetic geometry, in E. M. FrIEDLANDER AND D. R. GraYson (EDITORS), Handbook of K-theory volume 1, Springer, 2005.

[KM] M. Kolster And A. Movahnedi, Galois Co-descent for Etale Wild Kernels and Capitulation, Ann. Inst. Fourier 50 n. 1 (2000), 35-65.

[Ko] M. Kolster, $K$-theory and Arithmetic, Contemporary developments in algebraic $K$-theory, ICTP Lect. Notes XV, Abdus Salam Int. Cent. Theoret. Phys., 2004, 191-258.

[Lem] F. Lemmermeyer, Class groups of dihedral extensions, Math. Nachr. CCLXXVIII (2005), 679-691.

[Li] S. Lichtenbaum, Values of zeta functions, étale cohomology and algebraic K-theory, in H. BASS (EDITOR), Algebraic K-theory II, Lecture Notes in Mathematics 342, Springer, 1973.

[Neu] J. Neukirch, The Beilinson conjecture for algebraic number fields in M. RAPOPORT, P. SchnEIDER AND N. SCHAPPACHER (EDITORS), Beilinson's conjectures on special values of L-functions, Perspectives in Mathematics 4, Academic Press, 1988.

[NSW] J. Neukirch, A. Schmidt, K. Wingberg, Cohomology of number fields, Grundlehren der mathematischen Wissenschaften 323, Springer-Verlag, 2000.

[So] C. Soulé, K-théorie des anneaux d'entiers des corps de nombres et cohomologie étale, Invent. Math. 55 (1979), 251-295.

[We] C. WeIBEL, The norm residue isomorphism theorem, J. Topol. no. 2 (2009), 346-372. 